Original article

\title{
The psychoemotional status and cardiovascular system functional state of the first-year students under the influence of examination stress
}

\author{
Liliana K. Tokaeva ${ }^{1}$, Svetlana S. Parshina ${ }^{1}$, S.S. Pavlenkovich ${ }^{2}$ \\ ${ }^{1}$ Saratov State Medical University n.a. V.I. Razumovsky, Saratov, Russia \\ ${ }^{2}$ Institute of Physical Culture and Sports, Saratov State University named after N. G. Chernyshevsky, Saratov, Russia
}

Received 25 August 2012, Revised 20 September 2012, Accepted 12 October 2012.

(C) 2012, Tokaeva L.K., Parshina S.S., Pavlenkovich S.S.

C 2012, Russian Open Medical Journal

\begin{abstract}
The aim of this research is to study of the influence of examination stress on psycho-emotional status and functional state of the cardiovascular system of the 1-st year students of pedagogical high school. Methods - The study involved 105 young men aged 17-18 enrolled in the specialty "Physical Education". The studies were conducted during the period in-between the exams and during the examination session. The psycho-emotional status was determined by the SAN test questionnaire and test and the CH.D. Spielberg test, adapted for Russia by Ju.L. Khanin. The state of CVS autonomic regulation was evaluated by heart rate, blood pressure, endurance ratio, Kerdo index and the adaptive capacities by P.M. Bayevsky. Results - In the absence of exposure to stress in the majority of young men the studied parameters are within normal limits, indicating sufficient adaptive capabilities. A clear correlation between the level of personal anxiety in students and the nature of their reactivity to examination stress was found: the higher the anxiety level in a student is, the more stress resistance decreases and more pronounced changes in the cardiovascular system autonomic regulation appear. The strain of adaptation mechanism was found in a stressful situation in the first-year students with a high level of personal anxiety and satisfactory adaptation - in young men with average and low personal anxiety.
\end{abstract}

Keywords: students, anxiety, cardiovascular system, examination stress, autonomic regulation.

Cite as Tokaeva L.K., Parshina S.S., Pavlenkovich S.S. The psychoemotional status and cardiovascular system functional state of the first-year students under the influence of examination stress. Russian Open Medical Journal 2012; 1: 0304.

Correspondence to Prof. Liliana K. Tokaeva. Address: Department of Physiology n.a. I.A. Chuevsky, Saratov State Medical University n.a. V.I. Razumovsky, 112, Bolshaya Kazachiya str., Saratov, 410012, Russia. E-mail: normal@yandex.ru.

\section{Introduction}

University students are a special social group. It is characterized by the specific work and life conditions. Learning process is a key factor in causing adaptive physiological adjustment the students' bodies, specifically in the cardiovascular system (CVS) [1]. Studying in a University is different from studying at school in its structure and conditions: the amount of assimilated knowledge increases, the intensity of mental work goes up, there is pronounced imbalance in the work load, it dramatically increases during tests and examinations, majors and new forms of learning monitoring and evaluation appear, changing the nature of teacher-student relationships. Permanent mental and psychoemotional stress, the violation of work, rest and eating regimes often lead to disrupting adaptation and developing a number of diseases among students. Students of the Physical Education Department tend to combine academic and sports activities, which reflects on their mental and emotional status.

Exam stress is one of the main causes of emotional stress in students. Examination excitement is a factor that prevents from passing an exam successfully [2], as fear blocks the intellect, paralyzes the will, impairs the memory and reduces the ability to concentrate. Besides, the period of preparation for examinations is characterized by an intense mental activity, a significant limitation of motor activity, changes in sleep patterns, emotional pressure. All this affects the mechanisms of regulation of bodily physiological systems [2-4], including the CVS.

The aim of our study was to investigate the influence of examination stress on the psycho-emotional status and the CVS autonomic regulation in the Physical Education Department students in the initial period of studying at the university.

\section{Material and Methods}

The study included 105 first-year students (100\% male) of the Physical Education Department of N.G. Chernyshevsky Saratov State University Pedagogical College. The students' psychoemotional status was determined by a SAN test questionnaire [5] and the CH.D. Spielberger's test [6] (State-Trate-Anxiety-Inventory (STAI)), adapted for Russia by Y. Khanin [7].

The SAN test questionnaire is a form that contains 30 pairs of words with opposite meanings, reflecting different aspects of health, activity and mood. The examinee's task was: first, to find in each pair the attribute that best describes his state at the time of the examination; second, to assess the attribute's severity on a seven point scale.

The CH.D. Spielberger and Ju.L. Hanin's test includes 40 propositional questions, 20 of them are designed to assess 
situational anxiety, and 20 of them are aimed at personal anxiety. For each judgment the examinee should choose one of four offered answers. Then the examiner determines the level of development of both anxiety types using a special answer key. The final score was calculated on a scale in points: less than 30 points means the low anxiety level, from 30 to 45 points means the average anxiety level, and more than 45 points means the high anxiety level.

The state of autonomic CVS regulation was assessed by the heart rate, the systolic (SBP), diastolic (DBP) and pulse (PP) blood pressure (PP $=$ SBP-DBP). The degree of CVS regulation potential was assessed by the endurance factor (EF), which was calculated by the Kvas formula [8]: $E F=(H R / P P) \bullet 100$. To characterize the vegetative status, the Kerdo autonomic index (KAI) was calculated by the formula [8]: KAI=(1-DBP/HR) $\bullet 100 \%$.

For a more complete description of bodily adaptive capabilities the adaptive potential (AP) of RM Baevsky was calculated by the formula [9]: $\mathrm{AP}=0.011 \bullet \mathrm{HR}+0.014 \bullet \mathrm{SBP}+0.008 \bullet \mathrm{DBP}+0.014 \bullet \mathrm{Age}+$ $0.009 \bullet$ Weight $-0.009 \bullet$ Height -0.27 . The evaluation of results: AP less than 2.59 points means a satisfactory adaptation, $2.60-3.09$ points means the strain of adaptative mechanisms, $3.10-3.49$ points means poor adaptation, 3.50 points and more means adaptation failure.

Research was conducted in two stages: Stage 1 - between sessions on a day of practical in anatomy, Stage 2 - on the anatomy exam day before getting the exam question.

The data was statistically processed by the Statistica - 5 software package. For each data group the mean value $(\mathrm{M})$ and the error of the mean $(m)$ were calculated. The significance of differences was assessed by the Student's t-test at $p<0.05$.

\section{Results}

At the first stage of the research, in accordance with the CH.D. Spielberger and Ju.L. Hanin's test, the physical education department students were divided into 3 groups according to the personal anxiety (PA) level: high (group 1), normal (group 2) and low (group 3) PA level (Table 1).

Group one consisted of $33.3 \%$ of the total surveyed first-year students with the average PA of $50.4 \pm 0.2$ points. Group two contained the majority (42\%) of the first year students (PA $37.4 \pm 0.3$ points). Group three included $24.7 \%$ of the young men with the average PA of $20.5 \pm 0.4$ points. Note that during the practical the majority $(75.2 \%)$ of students were registered with the average reactive (situational) anxiety (RA) and $24.8 \%$ - with the low RA level.

By the SAN test results, the majority of the young men from group one state that their condition is below average, and their activity and mood are average. Boys in group 2 mostly have average SAN values. Students in group 3 have the subjective assessment of all SAN parameters higher when compared with the high and medium PA: health and activity above average, and high mood $(p<0.05)$. The 'mood' parameter was concluded to get higher evaluation from students in all groups than "health" and "activity" (Table 1).

In assessing the base CVS state with no exposure to stress, the majority of young men had heart rate and blood pressure rates within the guideline values for this age group (Table 1). However, young men in group 1 had heart rate significantly higher in comparison with peers in groups 2 and $3(p<0.05)$. In blood pressure no significant differences were seen. The mean value of
$E F$ in the first-years in all groups indicates sufficient functional potential of the circulatory system. In assessing neurovegetative status in all groups, most young men revealed severe vagotony, as evidenced by the average negative KAl. Sympathicotonia and eutonia were found in isolated cases. Adaptive potential points to the satisfactory state of adaptation mechanisms.

Table 1. Effect of examination stress on the psycho-emotional status and functional state of CVS in first-year students with different levels of anxiety $(\mathrm{M} \pm \mathrm{m})$

\begin{tabular}{|c|c|c|c|c|}
\hline \multirow{2}{*}{ Characteristics } & \multirow{2}{*}{$\begin{array}{c}\text { Research } \\
\text { Stage }\end{array}$} & \multicolumn{3}{|c|}{ Anxiety Level } \\
\hline & & High $(n=35)$ & Average $(n=44)$ & Low $(n=26)$ \\
\hline \multirow[t]{2}{*}{ PA, points } & 1 & $50.4 \pm 0.2$ & $36.3 \pm 0.7^{\infty}$ & $19.9 \pm 0.4^{\infty}$ \\
\hline & 2 & $51.0 \pm 0.5$ & $37.1 \pm 0.6^{\infty}$ & $20.9 \pm 0.3^{\infty}$ \\
\hline \multirow[t]{2}{*}{ RA, points } & 1 & $46.2 \pm 0.6$ & $35.0 \pm 0.6^{\infty}$ & $18.8 \pm 0.6^{\infty}$ \\
\hline & 2 & $61.4 \pm 0.09 *$ & $46.5 \pm 0.6^{*^{\infty}}$ & $31.3 \pm 0.8^{*^{\infty}}$ \\
\hline \multirow{2}{*}{$\begin{array}{l}\text { Condition, } \\
\text { points }\end{array}$} & 1 & $2.9 \pm 0.05$ & $4.7 \pm 0.06^{-\infty}$ & $5.73 \pm 0.04^{\infty}$ \\
\hline & 2 & $2.7 \pm 0.05^{*}$ & $4.5 \pm 0.06^{*^{\infty}}$ & $5.41 \pm 0.04^{*^{\infty}}$ \\
\hline \multirow{2}{*}{$\begin{array}{l}\text { Activity, } \\
\text { points }\end{array}$} & 1 & $3.3 \pm 0.02$ & $4.8 \pm 0.06^{\infty}$ & $5.72 \pm 0.04^{\infty}$ \\
\hline & 2 & $3.2 \pm 0.02 *$ & $4.68 \pm 0.06^{\infty}$ & $5.54 \pm 0.04^{*}$ \\
\hline \multirow[t]{2}{*}{ Mood, points } & 1 & $3.5 \pm 0.05$ & $5.2 \pm 0.06^{\infty}$ & $6.3 \pm 0.04^{\infty}$ \\
\hline & 2 & $3.4 \pm 0.05$ & $4.96 \pm 0.06^{*}$ & $5.92 \pm 0.04^{*}$ \\
\hline \multirow[t]{2}{*}{$\mathrm{HR}$, bits/min } & 1 & $71.1 \pm 0.4$ & $67.1 \pm 0.3^{\infty}$ & $62.5 \pm 0.3^{\infty}$ \\
\hline & 2 & $97.1 \pm 0.5^{*}$ & $88.1 \pm 0.5^{*^{\infty}}$ & $82.8 \pm 0.5^{*^{\infty}}$ \\
\hline \multirow[t]{2}{*}{$\mathrm{SBP}, \mathrm{mm} \mathrm{Hg}$} & 1 & $119.7 \pm 0.4$ & $117.2 \pm 0.5$ & $114.3 \pm 0.5^{\infty}$ \\
\hline & 2 & $142.6 \pm 1.02 *$ & $135.0 \pm 0.3^{*}$ & $133.0 \pm 0.7^{* \infty}$ \\
\hline \multirow[t]{2}{*}{$\mathrm{DBP}, \mathrm{mm} \mathrm{Hg}$} & 1 & $75.9 \pm 0.5$ & $75.1 \pm 0.5$ & $72.9 \pm 0.4^{\infty}$ \\
\hline & 2 & $86.4 \pm 0.6^{*}$ & $83.6 \pm 0.4^{*}$ & $80.2 \pm 1.0 *^{\infty}$ \\
\hline \multirow[t]{2}{*}{$\mathrm{PP}, \mathrm{mm} \mathrm{Hg}$} & 1 & $43.9 \pm 0.7$ & $42.0 \pm 0.6$ & $41.3 \pm 0.5$ \\
\hline & 2 & $56.4 \pm 0.4^{*}$ & $51.4 \pm 0.4^{*}$ & $52.9 \pm 0.7 *$ \\
\hline \multirow[t]{2}{*}{ EF, c.u. } & 1 & $16.5 \pm 0.2$ & $16.5 \pm 0.3$ & $15.4 \pm 0.2^{\infty}$ \\
\hline & 2 & $17.4 \pm 0.2 *$ & $17.2 \pm 0.7$ & $15.8 \pm 0.3^{\infty}$ \\
\hline \multirow[t]{2}{*}{$\mathrm{KAI}, \%$} & 1 & $-7.06 \pm 0.2$ & $-12.4 \pm 0.2^{\infty}$ & $-17.2 \pm 0.3^{\infty}$ \\
\hline & 2 & $10.9 \pm 0.9 *$ & $4.97 \pm 0.5^{*^{\infty}}$ & $-4.0 \pm 1.0^{*}$ \\
\hline \multirow[t]{2}{*}{ AP, points } & 1 & $2.09 \pm 0.01$ & $2.0 \pm 0.01^{\infty}$ & $1.87 \pm 0.01^{\infty-}$ \\
\hline & 2 & $2.78 \pm 0.02^{*}$ & $2.55 \pm 0.02^{*^{\infty}}$ & $2.03 \pm 0.01^{\infty}$ \\
\hline
\end{tabular}

1 - The day of the practical, $2-$ Before the exam.

$*$ is significant difference $(p<0.05)$ from the practical day. ${ }^{\infty}$ is significant difference $(p<0.05)$ from students with high PA level.

At stage 2 of the study we found that the students's psychoemotional stress peaks before taking the exam task, which affects the anxiety values and the functional CVS status.

Thus, in most boys from groups 1 and 2 a high RA level was recorded. Moreover, students with high AT have a stronger reaction to the pre-exam situation, as evidenced by significantly higher rates of RA compared with the group 2 students $(p<0.05)$. The first-years from group 3 had the average RA. In addition, in all groups was the common trend in the deterioration of psychoemotional status of the parameters "condition", "activity" and "mood" compared to the values, recorded in the period inbetween the exam sessions. However, students from groups 1 and 2 rate their health, activity and mood as average, whereas boys from group three mostly give the "above average" evaluations (Table 1).

When the students are waiting for the exam, there is the common trend to increased hemodynamic parameters. However, there have also been some differences in the CVS reactivity in students with different PA, the majority of which were credible $(p<0.05)$. Thus, students with the high PA had the heart rate increased by $36.6 \%$, those with the average PA had it increased by $31.3 \%$ and those with the low PA had it increased by $32.2 \%$. In comparison to the period in-between the exam sessions, the students had certain changes in blood pressure values before the 
exam. Moreover, the most pronounced changes were in the high PA students. Changing KIA was indicative for characterizing PA responses to stress in the young men, its values increased in the students who were waiting for the exam, regardless of the PA level. Significant differences in the groups are also found in the AP parameter. The mean AP value indicates stressed adaptation mechanisms in all first-years with high PA. At the same time, the young men with average and low PA have, on the country, satisfactory adaptation to a stressful situation (Table 1). However, an individual assessment of this parameter showed stressed regulation mechanisms in $29.5 \%$ of boys with a mean PA (AP equals $2.67 \pm 0.02$ points).

\section{Discussion}

One of the factors, that determine the first years' adapting to the university studies, is an adaptation to stressful situations, mainly to psycho-emotional stress, especially during exams [2]. The reactivity of a student's body to exam stress depends on the stress factor, the student's personal characteristics, as well as the speed, at which he is getting adjusted to the university studies.

In our study psycho-emotional stress in the situation of waiting for an exam led to a significant increase in the reactive anxiety level, primarily in the first year students with high personal anxiety. Several cardiovascular system indicators were shifted in the form of increased heart rate, BP, KIA and AP values, maybe due to the activation of regulatory mechanisms of sympathetic segment of the vegetative nervous system [2,4]. More significant changes in hemodynamics values in students with high personal anxiety indicate a low level of adaptation to stressful situations [3]. All first-year students with the low PA and most young men with the average PA, in contrast, had a milder adaptation to the exam stress.

\section{Conclusion}

Forming stress resistance is a key factor for students' mental health, ensuring successful and reliable academic and professional activity. High anxiety level, as well as the high degree of mental stress contribute to developing reduced stress resistance in academic activity. In this regard it is necessary to substantiate the ways, methods and guidelines for overcoming stress states in the process of personal and professional development of future physical education specialists.

Conflict of interest: none declared.

\section{Reference}

1. Sitdikov FG, Shaikhelislamova MV, Valeev IR. The Effect of Academic Load and Work Conditions on the Sympathoadrenal Function and the Indices of Heart Rhythm Regulation in 17- to 18-Year-Old Girls. Human Physiology 2001; 27(5): 561-567 (doi: 10.1023/A:1011908310303).

2. Shcherbatykh luV. Effects of parameters of the higher nervous activity on stress in students during exams. Zh Vyssh Nerv Deiat Im I P Pavlova (I.P. Pavlov Journal of Higher Nervous Activity) 2000; 50(6): 959-965 (PMID: 11190095) [Article in Russian].

3. Yumatov EA, Kuzmenko VA, Badikov VI, Glazachev OS, Ivanova LI. Emotional Stress in Students during Examinations. Human Physiology 2001; 27(2): 221-227 (doi: 10.1023/A:1011039702932).

4. Schepard JD, Al'Absi M, Whitsett TL, Passey RB, Lovallo WR. Additive pressor effects of caffeine and stress in male medical students at risk for hypertension. Am J Hypertens 2000; 13: 475-481 (PMID: 10826397).

5. Doskin VA, Lavrentyeva NA, Miroshnikov MP, Sharai WB. Differentiated functional state self-test. Problems of Psychology 1973 (6): 141-145 [Article in Russian].

6. Spielberger CD, Gorsuch RL, Lushene R. Manual for State-Trait Anxiety Inventory. Consulting Psychologist Press, California, USA, 1970.

7. Karelin A. Great Encyclopedia of psychological tests. Eksmo Publ., Moskow, Russia, 2007. 416 p. [Text in Russian]

8. Dubrovsky V.I. Valeology. Healthy Lifestyle. Retorika Publ., Moskow, Russia, 2001. 560 p. [Text in Russian]

9. Bayevsky R.M., Berseneva A.P. Evaluation of adaptive capacity and the risk of disease. Medicine Publ., Moskow, Russia, 1997. 236 p. [Text in Russian]

\section{Authors:}

Liliana K. Tokaeva - MD, D.Sc., Professor, Department of Normal Physiology n.a. I.A. Chuevsky, Saratov State Medical University n.a. V.I. Razumovsky, Saratov, Russia;

Svetlana S. Parshina - MD, D.Sc., Professor, Department of Therapy, Faculty training and retraining of specialits, Saratov State Medical University n.a. V.I. Razumovsky, Saratov, Russia;

S.S. Pavlenkovich - Senior Lecturer, Department of the theoretical foundations of physical education, Institute of Physical Culture and Sports, Saratov State University named after N. G. Chernyshevsky, Saratov, Russia. 